\title{
UM MODELO OTIMIZADOR PARA ORGANIZAÇÃO DOS COMPARTIMENTOS DE EXPLORAÇÃO EM FLORESTA TROPICAL ${ }^{1}$
}

\author{
Evaldo Muñoz Braz² ${ }^{2}$ Celso Carnieri ${ }^{3}$ Julio Eduardo Arce ${ }^{4}$
}

\begin{abstract}
RESUMO - Existe a necessidade de garantia de um fluxo constante de madeira com potencial comercial para a viabilidade da pequena propriedade, porém nem sempre isto é possível, pelo fato de os talhões serem pequenos e, principalmente, a distribuição das espécies de interesse na floresta nativa ser irregular, por serem os ecossistemas heterogêneos e pela variedade de tipologias florestais que formam um complexo mosaico. Este trabalho teve como finalidade principal apresentar um modelo matemático que auxilie no planejamento e na distribuição dos talhões em uma pequena propriedade da floresta tropical. O modelo matemático resultou em um novo conceito de talhão. Com base no inventário pré-exploratório, os talhões foram subdivididos em compartimentos, onde foram consideradas características diferenciais como: espécie, volume por espécie, abundância, possibilidade de mercado e, principalmente, valor final de cada subtalhão. Para definição do modelo matemático utilizou-se a PM (programação por metas), que se faz necessária na seleção dos subtalhões, combinando-os e unindo-os em uma área equivalente ou menor que a do compartimento anual original. Este modelo, ao mesmo tempo que organiza novos talhões, determina a taxa de extração anual e sua possível renda, além de limitar a área do compartimento a ser explorado, o que se faz necessário para garantia da regeneração do compartimento no ciclo de corte previsto. Como resultado, o modelo proposto mostrouse bastante superior ao formal e garantiu sobremaneira o equilíbrio do rendimento anual para o pequeno proprietário.
\end{abstract}

Palavras-chave: Manejo florestal, ecossistemas heterogêneos, modelo matemático, programação por metas e subtalhões.

\section{AN OPTIMIZING MODEL FOR ORGANIZING HARVESTING COMPARTMENTS IN TROPICAL FOREST MANAGEMENT}

\begin{abstract}
There is a need to ensure an even flow of timber with commercial potential to make forest management viable on small farms; however, this need is not always met, due to the fact that compartments are small and especially due to the irregular distribution of the species of interest in the native forest because of the heterogeneous ecosystems and forest typologies that form a complex mosaic. The main purpose of this work was to develop a mathematical model to help with the planning and distribution of stands in a small tropical forest farm. The mathematical model resulted in a new concept of stand. Based on the pre-exploratory forest inventory, the compartments were divided into sub compartments with their different values being calculated according to market timber prices. Since the annual logging rate allowed had already been defined based on local and regional studies, the goal was to guarantee that this rate, transformed in monetary value can really be reached annually. The mathematical model GP (Goal programming) was utilized in the selection of the sub compartments by grouping them into a final compartment with size similar to that of the original annual compartment. The proposed stand model was compared with the type of stand normally used in tropical forests and was proved rather superior to the conventional one, thus guaranteeing small farmers a balanced annual income.
\end{abstract}

Keywords: Forest management, heterogeneous ecosystems, mathematical model, goal programming, sub compartments.

1 Recebido para publicação em 24.4.2002 e aceito para publicação em 17.02.2004.

2 Engenheiro Florestal, pesquisador, M.S. em Manejo Florestal, Embrapa Florestas, Estrada da Ribeira, Km 111, Caixa Postal 319, 83411-000 Colombo-PR, <evaldo@cnpf.embrapa.br>. ${ }^{3}$ Professor do Dep. de Matemática da Universidade Federal do Paraná UFPR, Doutor na área de Pesquisa Operacional, <carnieri@ mat.ufpr.br>. ${ }^{4}$ Doutor em Ciências Florestais, Manejo Florestal, Dep. de Ciências Florestais - UFPR, <jarce@ floresta.ufpr.br>. 


\section{INTRODUÇÃO}

O volume madeireiro de um povoamento nativo varia em função da capacidade produtiva do solo e da distribuição irregular das espécies. Logo, é impossível esperar que uma divisão da área em partes iguais vá corresponder à divisão do volume também em partes iguais. Por isto, em cada ano as produções volumétricas ou concentrações de espécies podem ser relacionadas com a área. Assim, os sítios de alta produtividade devem ter áreas menores e os de baixa, áreas maiores (Hosokawa et al., 1998). No manejo florestal, é necessário garantir um fluxo constante de madeira com potencial comercial para que ele seja viável, principalmente quando se trata de pequena propriedade. Porém, nem sempre isto é possível, devido ao pequeno tamanho dos talhões e à distribuição irregular das espécies de interesse na floresta nativa, ou seja, mesmo que o crescimento do povoamento como um todo seja regular e de acordo com os estudos de crescimento efetuados, pelo menos a curto prazo a alocação dos talhões para garantir quantidades homogêneas é praticamente impossível dentro dos critérios atuais. Tal fato ocasiona que se em um ano a renda da madeira pode ser satisfatória, no ano seguinte pode ser aquém do idealizado pelo produtor ou pelo plano original. Além disto, com relação a talhões com excelente estoque, o que aparentemente garantiria uma renda acima do esperado em determinado ano, estes ficam limitados por quatro questões básicas: a) existe um limite de extração por talhão definido no IBAMA (1998) pelo Plano de Manejo previsto para a área, não podendo este valor ser ultrapassado; b) existe um ciclo de corte a ser respeitado, segundo critérios do IBAMA, e novas espécies podem entrar no mercado inesperadamente, e se limitadas em um talhão maior que já foi explorado, só se tornarão aptas para a venda no próximo ciclo, podendo inviabilizar a renda anual do pequeno proprietário; c) existe a carência de mão-de-obra, o que limita extrações acima do previsto anteriormente pelo plano; e d) pode-se ainda acrescentar uma quarta limitante diretamente ligada à anterior: os manejadores destas pequenas propriedades são primeiramente agricultores, o que significa pouco tempo livre para outras atividades, como roçado, limpeza do terreno, plantio, entre outras.

O modelo proposto visa especificamente possibilitar uma renda anual equilibrada da floresta, com base em seu potencial médio avaliado em inventário, mediante um novo modelo de compartimentos compostos. Este novo compartimento é formado por subcompartimentos agrupados através da PM (programação por metas), visando equilibrar as distorções que os sítios de diferentes potenciais em madeira ocasionam. Assim, os novos compartimentos compostos terão áreas diferentes, mas valor final semelhante. $O$ ganho principal para os pequenos proprietários é a garantia de uma renda semelhante a cada ano, eliminando rendas distorcidas ou mesmo inexistentes em determinado ano, devido a compartimentos mais "fracos" por falta de árvores de valor econômico.

\section{MATERIAL E MÉTODOS}

\subsection{Características da Área}

O experimento foi desenvolvido no Projeto de Colonização Pedro Peixoto, implantado pelo INCRA (Instituto Nacional de Colonização e Reforma Agrária), no município de Senador Guiomar, Estado do Acre, na propriedade de Alfredo Ferreira Rodrigues, BR 364, $\mathrm{Km} 80$.

O clima é do tipo Aw (Köppen), com três meses de período seco. A precipitação anual varia de 1.800 a $2.000 \mathrm{~mm}$ e a temperatura média anual é de $24^{\circ} \mathrm{C}$. O período de estiagem vai de junho a setembro, o que permite que as atividades de exploração florestal sejam executadas até o início de outubro, quando se iniciam as primeiras chuvas. Os solos predominantes são os Latossolos Vermelho-Amarelos distróficos. A vegetação predominante é de floresta tropical semiperenifólia, com formações de floresta aberta e floresta densa. A parte nãoflorestal é basicamente formada por pastagens, culturas de subsistência e algumas culturas perenes (Brasil, 1976). A floresta manejada possui volume total médio (DAP > $10 \mathrm{~cm}$ ) de $180,0 \mathrm{~m}^{3} / \mathrm{ha}$ e volume total de madeira de espécies com mercado para consumo interno e exportação de $42,5 \mathrm{~m}^{3} /$ ha (Oliveira, 2000). Nesta parte aparecem espécies madeireiras de valor econômico como angelim, cedro e cerejeira. A parte não-florestal é formada por pastagens e culturas de subsistência, com poucas culturas perenes como café.

As áreas de manejo são cortadas pelos afluentes do igarapé Iquiri, formando vários pequenos igarapés, na sua maioria semiperenes. A topografia predominante é plana, com declividades em torno de $5 \%$ e quase nunca superiores a $10 \%$.

Os lotes de Pedro Peixoto têm em torno de 80,0 ha (400 x $2.000 \mathrm{~m})$ cada. 


\subsection{Modelo Atual}

O modelo apresentado a seguir é o utilizado por todas as 20 propriedades na área que realizam manejo florestal, tendo periodicamente dificuldades por verificar, muitas vezes, que em determinado ano o compartimento não proporcionará renda devido à falta de espécies de valor.

Cada propriedade possui dez compartimentos. Como o tamanho-padrão dos lotes é em torno de 80 ha e a parte destinada à Reserva Legal de 40 ha, cada compartimento tem cerca de 4,0 ha, ou seja, são explorados, a cada ano, 4 ha de floresta em cada propriedade. Estes compartimentos possuem tamanho igual, mas rendas potenciais bem diferentes.

Nesse modelo, o ciclo é de dez anos e a intensidade de corte de $40 \mathrm{~m}^{3} /$ compartimento.

\subsection{Modelo Proposto}

No modelo atual a área de manejo é dividida, como já exposto, em dez compartimentos. No modelo proposto estes dez compartimentos serão subdivididos em quatro cada um, formando um total de 40 subcompartimentos.

O modelo proposto combinará, então, os subcompartimentos de acordo com seu potencial em espécies de valor, formando um novo talhão ideal que proporcione a renda desejada (Braz, 2001). Os novos compartimentos compostos terão agora tamanho variável, mas rendas semelhantes.

O ciclo e a intensidade de corte serão também de dez anos e $40 \mathrm{~m}^{3} /$ compartimento.

\subsubsection{Subcompartimentalização}

Com base no inventário pré-exploratório, os talhões serão subdivididos em compartimentos menores de tamanho igual, considerando a distribuição das espécies comerciais, o volume, a abundância e o valor.

\subsubsection{Estudo do Modelo}

Como a taxa anual máxima já é definida pelo IBAMA, com base em estudos anteriores locais (Araújo \& Oliveira, 1996; Oliveira \& Braz,1998; Oliveira, 2000) e regionais (Silva, 1993), a meta é garantir que esta taxa, transformada em valor monetário, realmente possa ser alcançada. Como já esclarecido anteriormente, isto é sempre dificultado pelo potencial não-homogêneo dos talhões. A taxa anual de exploração de madeira foi expressa, baseando-se no valor das diferentes espécies por grupo diferenciado de valor, no porcentual permitido de extração por classe de espécie/diâmetro e no dano mínimo estabelecido ao povoamento. A taxa de extração anual deverá ser sustentável, ou seja, dentro dos incrementos previstos em pesquisas realizadas na Amazônia e principalmente no Estado do Acre.

Para esses cálculos será utilizada a programação por metas (PM), uma extensão da programação linear. A PM corrige as limitações da PL, forçando na direção de todos os objetivos simultaneamente, tratando todas as metas da mesma maneira e dando também (se necessário) diferentes pesos a elas (Buongiorno \& Gilles, 1987).

A PM minimiza o desvio de múltiplas metas, ou objetivos, sujeitas a algumas restrições, que são metas determinadas, sendo outras restrições físicas (Dykstra, 1984).

O modelo proposto, ao mesmo tempo que organiza novos talhões, informa indiretamente a taxa de extração anual e sua possível renda e define a área do compartimento a ser explorado. Isto se faz necessário para garantia da regeneração do compartimento no ciclo de corte previsto.

Dentro das restrições previstas, a função-objetivo buscará a maximização da renda anual.

No caso deste trabalho, deseja-se obter rendas anuais em torno de US\$ 2,000.00 (no momento do levantamento dos dados, equivalente à extração de $40 \mathrm{~m}^{3} / \mathrm{ano} /$ talhão), durante um período de dez anos. Nossas metas anuais, portanto, não podem ser fixas, mas flexíveis. Para isto são utilizados os recursos de desvios de excesso ou de falta, minimizando-os.

As restrições básicas serão que a área de cada subtalhão só pode ser cortada uma vez a cada dez anos, devido ao ciclo de corte anteriormente definido, e o somatório da renda dos subtalhões deverá estar em torno de US\$2,000.00.

\subsubsection{Cálculo do Valor da Madeira de cada Subtalhão}

As árvores consideradas foram somente aquelas que podem ser extraídas sem ameaçar a estrutura posterior da floresta, para garantia de outros ciclos. Cada árvore de

R. Árvore, Viçosa-MG, v.28, n.1, p.77-83, 2004 
cada talhão teve seu volume final de madeira já transformada calculado segundo Araújo (1998), para essa mesma área.

Esse volume foi multiplicado pelo valor de cada espécie no mercado de Rio Branco. Os resultados por subtalhão aparecem no Quadro 1 (renda bruta proporcionada pelas espécies comerciais imediatas).

\subsubsection{Função-Objetivo}

A função-objetivo deverá minimizar os desvios da renda bruta, visando obter uma renda semelhante anualmente de acordo com a reunião de subtalhões.

As variáveis $d$ e $e$, segundo a falta ou o excesso, respectivamente, tentam adequar o existente à meta desejada.

$$
\operatorname{Min} Z=\sum_{i=1}^{10} d_{i}+\sum_{i=1}^{10} e_{i}
$$

em que $i=$ talhão.

\subsubsection{Restrições}

As restrições de corte e renda mínima (com base no potencial de extração da floresta previamente calculado) garantirão a nova estrutura dos talhões, com base no agrupamento adequado dos subtalhões.

\section{a) Restrições quanto ao corte de cada subtalhão}

Cada subtalhão deverá ser cortado somente uma vez durante o ciclo inteiro de dez anos, o que fica especificado na seguinte restrição:

$$
\sum_{k=1}^{10} X_{i j k}=1
$$

em que

$i=1, \ldots .10, j=1, \ldots .4 ; i=$ talhão; e $j=$ subtalhão.

\section{b) Restrições quanto à renda mínima anual}

A restrição principal é que a renda anual deverá ser compatível com os $40 \mathrm{~m}^{3} /$ compartimento permitidos de extração (devido a levantamentos e estudos anteriores), que renderão $20 \mathrm{~m}^{3} /$ compartimento devido a perdas na transformação de $50 \%$. Como em média o valor do metro cúbico transformado obtido no mercado de Rio Branco é de US\$100, 00, espera-se uma renda mínima anual de US\$ 2,000.00.

$$
\sum_{i=1}^{10} \sum_{i=1}^{4} V_{i j} \cdot X_{i j k}+d_{k}-e_{k}=2000, \quad k=1, \ldots, 10
$$

em que $d_{k}=$ desvio da meta por déficit no ano $\mathrm{k}$ (US\$); $e_{k}=$ desvio da meta por excesso no ano $k$ (US $\$$ ); $X_{i j k}=1$, se o subtalhão $j$ do talhão $i$ for explorado no ano $k$ e 0 , caso ocorra o contrário; e $V_{i j}=$ renda do subtalhão $j$ do talhão $i$ (US\$).

\section{RESULTADOS E DISCUSSÃO}

\subsection{Potencial de Cada Subtalhão}

Com as fórmulas anteriores, o processo e as espécies selecionadas, foi calculado o valor de cada talhão de acordo com o Quadro 1.

No Quadro 1 estão os volumes totais de cada subcompartimento, os volumes comerciais e a renda bruta esperada desta floresta. Como pode ser observado, os subcompartimentos, quando comparados entre si, são bastante heterogêneos. Existem oito subcompartimentos com valor 0 (zero). Este fato, sem dúvida, confirma a heterogeneidade da floresta tropical e, portanto, desequilibra a renda anual esperada. Também pode ser observado que apenas quatro dos talhões inteiros, em um total de dez, não apresentam este problema, o que vem a confirmar a necessidade de planejar melhor os talhões em floresta tropical.

\subsection{Resultado do Modelo Segundo Compartimento Formal}

De acordo com o Quadro 2 , observa-se que o modelo formal (padrão utilizado normalmente) apresenta as seguintes deficiências:

- 70\% dos talhões obtêm valores inferiores a US $\$ 2,000.00$ por ano.

- Somente três talhões alcançam e excedem o valorlimite, porém, por motivos de limitações da taxa anual de extração comprometida no IBAMA, com base nas taxas de crescimento encontradas, mantêm-se como valor máximo de extração US $\$ 2,000.00$, o que significa que o excesso de um talhão, neste modelo, não pode auxiliar o déficit de algum talhão posterior.

- Estes valores encontram-se, em média, 38\% abaixo do valor pretendido e, portanto, muito abaixo da capacidade da floresta. 
Quadro 1 - Distribuição da área de manejo em talhão e subtalhão, considerando volume total, volume comercial imediato e renda bruta relativa ao volume comercial imediato

Table 1 - Management area distribution into compartments and sub-compartments, considering total volume; immediate commercial volume and gross income relative to the immediate commercial volume

\begin{tabular}{|c|c|c|c|c|c|c|c|c|c|}
\hline Talhão 10 & Talhão 9 & Talhão 8 & Talhão 7 & Talhão 6 & Talhão 5 & Talhão 4 & Talhão 3 & Talhão 2 & Talhão 1 \\
\hline \multicolumn{10}{|c|}{ Ano 1 a 10} \\
\hline Sub 10.4 & Sub. 9.4 & Sub. 8.4 & Sub. 7.4 & Sub. 6.4 & Sub. 5.4 & Sub. 4.4 & Sub. 3.4 & Sub. 2.4 & Sub 1.4 \\
\hline $161,86^{1 /}$ & $27,4135 /$ & $21,8612 /$ & $106,986 /$ & $71,892 /$ & $43,4538 /$ & 92,9112 & $63,233 /$ & $57,2109 /$ & $46,8720 /$ \\
\hline$\overline{19,471}$ ㄴ & 0,000 & 3,8061 & 20,0864 & 19,0026 & 2,3231 & 19,030 & 3,6966 & 2,1484 & 7,6122 \\
\hline$\underline{1051,44^{3 /}}$ & 0,00 & 205,53 & $1.084,67$ & 1026,14 & 125,45 & $1.027,62$ & 199,62 & 116,01 & 411,06 \\
\hline Sub 10.3 & Sub. 9.3 & Sub. 8.3 & Sub. 7.3 & Sub. 6.3 & Sub. 5.3 & Sub. 4.3 & Sub. 3.3 & Sub. 2.3 & Sub 1.3 \\
\hline $75,7680 /$ & $60,3097 /$ & $65,5836 /$ & $60,1794 /$ & $71,800 /$ & $31,3833 /$ & $72,823 /$ & $42,1554 /$ & $81,2970 /$ & $91,140 /$ \\
\hline 0,0000 & 3,8000 & 3,8000 & 0,000 & 8,0959 & 11,4180 & 6,0721 & 0,0000 & 12,6884 & 19,0303 \\
\hline 0,00 & 205,20 & 205,20 & 0,00 & 437,18 & 616,57 & 327,89 & 0,00 & 685,17 & $1.027,64$ \\
\hline Sub 10.2 & Sub. 9.2 & Sub. 8.2 & Sub. 7.2 & Sub. 6.2 & Sub. 5.2 & Sub. 4.2 & Sub. 3.2 & Sub. 2.2 & Sub 1.2 \\
\hline $86,100 /$ & $68,5337 /$ & $57,3857 /$ & $66,866 /$ & $93,4506 /$ & $41,0397 /$ & $42,6889 /$ & $78,2886 /$ & $72,2667 /$ & 39,060 \\
\hline 25,4666 & 4,4587 & 7,6120 & 11,1775 & 7,9161 & 2,3587 & 10,4699 & 5,0430 & 11,4183 & 3,2770 \\
\hline $1.375,20$ & 240,77 & 411,05 & 603,59 & 427,47 & 127,37 & 565,37 & 272,32 & 616,59 & 176,96 \\
\hline Sub 10.1 & Sub. 9.1 & Sub. 8.1 & Sub. 7.1 & Sub. 6.1 & Sub. 5.1 & Sub. 4.1 & Sub. 3.1 & Sub. 2.1 & Sub 1.1 \\
\hline $110,208 /$ & $70,0164 /$ & $106,573 /$ & $86,9258 /$ & $143,784 /$ & $33,7974 /$ & $42,700 /$ & $93,3441 /$ & $66,0010 /$ & $54,684 /$ \\
\hline 49,4235 & 0,000 & 4,4587 & 0,000 & 45,5869 & 11,4761 & 0,0000 & 0,0000 & 20,2803 & 0,0000 \\
\hline $2.668,87$ & 0,000 & 240,76 & 0,00 & $2.461,69$ & 619,71 & 0,00 & 0,00 & $1.095,14$ & 0,00 \\
\hline
\end{tabular}

${ }^{1 /}$ Volume total do subtalhão, considerando DAP acima de $40 \mathrm{~cm}$ de espécies comerciais e não-comerciais. (Total Volume in the sub compartment considering DBH above $40 \mathrm{~cm}$ and commercial species and non commercial species).

2l Volume das espécies comerciais imediatas e com DAP acima de $50 \mathrm{~cm}$. (Immediate Commercial Species Volume with DBH above $50 \mathrm{~cm}$ ).

${ }^{3 /}$ Renda bruta proporcionada pelas espécies comerciais imediatas. (Gross income originated by immediate commercial species).

Quadro 2 - Retorno esperado de cada tal hão no modelo formal

Table 2 - Expected income of each compartment according to the conventional model

\begin{tabular}{|c|c|c|c|c|c|c|c|c|c|}
\hline Talhão 10 & Talhão 9 & Talhão 8 & Talhão 7 & Talhão 6 & Talhão 5 & Talhão 4 & Talhão 3 & Talhão 2 & Talhão 1 \\
\hline 5095,51 & $* 445,97$ & $* 1062,54$ & $* 1688,26$ & 4352,48 & $* 1489,1$ & $* 1920,88$ & $* 471,94$ & 2512,91 & $* 1615,66$ \\
\hline
\end{tabular}

* = valor abaixo do desejado (US\$2,000.00).

\subsection{Resultado do Modelo Considerando Compartimento Composto}

A programação por metas indicou o seguinte agrupamento dos subtalhões, ou seja, talhões compostos (Quadro 3).

Os déficits (D) ou excessos (E) anuais encontrados foram:
D2
32,69

D3

53,42

$\begin{array}{lr}\text { D4 } & 13,86 \\ \text { D5 } & 54,95 \\ \text { D7 } & 8,20 \\ \text { D8 } & 66,34 \\ \text { D9 } & 1,00 \\ \text { D10 } & 63,32 \\ \text { E1 } & 668,90 \\ \text { E6 } & 461,70\end{array}$

R. Árvore, Viçosa-MG, v.28, n.1, p.77-83, 2004 
Quadro 3 - Agrupamento de subtal hões

Table 3 - Sub compartment grouping

\begin{tabular}{|c|c|c|c|}
\hline Subtalhão & $\begin{array}{c}\text { Novo } \\
\text { Agrupamento }\end{array}$ & Subtalhão & $\begin{array}{c}\text { Novo } \\
\text { Agrupamento }\end{array}$ \\
\hline $\mathrm{X} 11$ & 6 & X61 & 6 \\
\hline $\mathrm{X} 12$ & 9 & X62 & 10 \\
\hline X13 & 3 & X63 & 8 \\
\hline X14 & 8 & X64 & 5 \\
\hline $\mathrm{X} 21$ & 4 & X71 & 4 \\
\hline $\mathrm{X} 22$ & 7 & $\mathrm{X} 72$ & 2 \\
\hline X23 & 4 & X73 & 5 \\
\hline X24 & 5 & $\begin{array}{l}\mathrm{X} 74 \\
\mathrm{X}\end{array}$ & 8 \\
\hline X31 & 10 & X81 & 10 \\
\hline $\mathrm{X} 32$ & 5 & X82 & 3 \\
\hline $\mathrm{X} 33$ & 4 & $\mathrm{X} 83$ & 9 \\
\hline X34 & 5 & X84 & 5 \\
\hline $\mathrm{X} 41$ & 5 & X91 & 1 \\
\hline $\mathrm{X} 42$ & 9 & X92 & 10 \\
\hline $\mathrm{X} 43$ & 3 & X93 & 4 \\
\hline $\mathrm{X} 44$ & 10 & X94 & 6 \\
\hline X51 & 2 & X101 & 1 \\
\hline X52 & 2 & $\mathrm{X} 102$ & 7 \\
\hline $\mathrm{X} 53$ & 2 & $\mathrm{X} 103$ & 9 \\
\hline $\mathrm{X} 54$ & 5 & X104 & 9 \\
\hline
\end{tabular}

- O déficit médio encontrado foi de $1,9 \%$ para o valor pretendido de US\$2,000.00, ou seja, praticamente negligenciável em comparação com o modelo anterior.

\subsection{Considerações Sobre os Modelos e as Limitações}

No Quadro 4 está a comparação dos dois modelos:

O modelo I é desaconselhável por não permitir a obtenção do potencial de retorno da floresta, principalmente por estar limitado a áreas com baixo volume comercial. Este modelo induz indiretamente à prática de ações ilegais, como explorar árvores potenciais de talhões já explorados ou que deverão ser explorados futuramente, para "completar" os estoques comerciais ideais.

Sem dúvida o modelo II garante a melhor distribuição de renda anual, dentro do previsto como limite da taxa de corte permitida de acordo com o potencial de cada talhão.

O modelo II organiza o melhor talhão inicial mediante um novo agrupamento de subtalhões (Braz, 2001). Deve-se observar que os números da ordem não significam uma ordem temporal de exploração dos talhões, e sim identifica talhões potencialmente semelhantes, podendo o manejador escolher a seqüência de talhões que melhor convier dentro dos critérios silviculturais.

A dispersão dos subtalhões pouco influirá nos custos e no planejamento, uma vez que se trata de pequenas propriedades, onde a maior distância entre eles não ultrapassa $1.000 \mathrm{~m}$.

O modelo pode ser rodado anualmente (no primeiro ciclo) com base em novas informações de espécies que entram no mercado (atualizando o modelo), dando maiores garantias de renda com a disposição das novas opções mais sustentáveis, econômica e ecologicamente.

Esse modelo possibilita a "economia" de áreas de subtalhões sem produção no primeiro ciclo, o que significa que não há necessidade de "fechar" esta área pelo período do ciclo de dez anos, uma vez que não há a preocupação com sua regeneração, pois não houve corte algum na área. No momento em que essa área, seja por ingresso, crescimento das árvores do talhão ou surgimento de novas espécies com possibilidades de mercado, tornar esse subtalhão viável, ele passa a ser considerado no modelo de otimização. No exemplo deste trabalho, nove subtalhões (ou seja, 22,5\% da área total) podem ser "poupados" para o momento mais adequado.

Quadro 4 - Comparação de modelos de tal hões Table 4 - Comparing both compartment models

\begin{tabular}{|l|c|c|c|c|c|c|c|c|c|c|}
\hline Talhão 10 & Talhão 9 & Talhão 8 & Talhão 7 & Talhão 6 & Talhão 5 & Talhão 4 & Talhão 3 & Talhão 2 & Talhão 1 \\
\hline 5095,51 & $* 445,97$ & $* 1.062,54$ & $* 1.688,26$ & $4.352,48$ & $* 1.489,1$ & $* 1.920,88$ & $* 471,94$ & $2.512,91$ & $* 1.615,66$ \\
\hline \multicolumn{8}{|c|}{ Modelo II (proposto) } \\
\hline $1.936,68$ & $1.999,00$ & $1.933,66$ & $1.991,80$ & $2.461,70$ & $1.945,05$ & $1.986,14$ & $1.946,58$ & $1.967,31$ & $2.668,00$ \\
\hline
\end{tabular}

R. Árvore, Viçosa-MG, v.28, n.1, p.77-83, 2004 
O que o modelo II pode fazer nada mais é que "indicar" talhões viáveis anuais, partindo do conceito renda, ou seja, ele define talhões mais homogêneos que os habitualmente existentes.

O modelo propicia o melhor direcionamento e aproveitamento dos tratamentos silviculturais, além de ter a vantagem, do ponto de vista ambiental, de poder fazer a exploração alternada dos subtalhões e talhões com ganhos para fauna, regeneração e recomposição das clareiras recém-formadas (distúrbios localizados) e diversidade biológica. Guardadas as proporções, este modelo pode também ser reproduzido para grandes talhões empresariais.

\section{CONCLUSÃO}

O modelo proposto garante, com vantagem, gerar um fluxo de rendimento anual equilibrado para o pequeno proprietário, o que facilita o planejamento anual de extração na pequena propriedade, podendo adequar-se para a otimização da produção madeireira em condições semelhantes na floresta tropical.

Conclui-se que o modelo possibilita o melhor talhão inicial, tornando possível no planejamento anual contar com o potencial médio identificado na floresta, segundo os levantamento apropriados.

\section{REFERÊNCIASBIBLIOGRÁFICAS}

ARAÚJO, H. J. B. Índices técnicos da exploração e transformação madeireira em pequenas áreas sob manejo florestal no PC. Pedro Peixoto, Acre: Embrapa Acre, 1998. p. 18. (Circular Técnica)

ARAUJO, H. J. B.; OLIVEIRA, L. C. Manejo florestal sustentado em áreas de reserva legal de pequenas propriedades rurais do PC. Pedro Peixoto-Acre. Rio Branco, AC: EMBRAPA-CPAF-AC, 1996, 7 p.

(EMBRAPA-CPAF-AC. Pesquisa em Andamento, 89).
BRAZ, E.M. Um modelo em programação linear para garantia do rendimento sustentado em pequena propriedade na floresta tropical. Curitiba: Universidade Federal do Paraná, 2001. 76 p.

BUONGIORNO, J.; GILLESS, J. K. Forest management and economics a primer in quantitative methods. New York: MacMillan Publishing Company, 1987. 89 p.

DYKSTRA, D. P. Mathematical programming for natural resource management. New York: McGraw-Hill Book Company, 1984. 319 p.

HOSOKAWA, R. T.; MOURA, J. B.; CUNHA, U. S. Introdução ao Manejo e Economia de Florestas. Curitiba: Universidade Federal do Paraná. 1998. 162 p.

IBAMA. Ministério do Meio Ambiente, dos Recursos Hídricos e da Amazônia Legal. Extensão do desflorestamento bruto no Brasil. Brasília: 1998. $150 \mathrm{p}$.

OLIVEIRA, M. V. N.; BRAZ, E. M. Manejo florestal em regime de rendimento sustentado, aplicado à floresta do Campo Experimental da Embrapa. Acre: 1998. 45 p. (Boletim de Pesquisa, 21)

OLIVEIRA, M. V. N. Sustainable forest management for small farmers in Acre State in the Brazilian Amazon. 2000. $133 \mathrm{f}$. Thesis (Ph.D.) - University of Aberdeen Soil and plant Science Department, 2000.

SILVA, J. N. M. Possibilidades para a produção sustentada de madeira em floresta densa de Terra-Firme da Amazônia Brasileira. Colombo: CNPF-EMBRAPA, 1993. 36 p. (Documento, 23) 